MATHEMATICS OF COMPUTATION

Volume 76, Number 259, July 2007, Pages 1141-1161

S 0025-5718(07)01973-4

Article electronically published on February 7, 2007

\title{
ROBUST NORM EQUIVALENCIES FOR DIFFUSION PROBLEMS
}

\author{
MICHAEL GRIEBEL, KARL SCHERER, AND MARC ALEXANDER SCHWEITZER
}

\begin{abstract}
Additive multilevel methods offer an efficient way for the fast solution of large sparse linear systems which arise from a finite element discretization of an elliptic boundary value problem. These solution methods are based on multilevel norm equivalencies for the associated bilinear form using a suitable subspace decomposition. To obtain a robust iterative scheme, it is crucial that the constants in the norm equivalence do not depend or depend only weakly on the ellipticity constants of the problem.

In this paper we present such a robust norm equivalence for the model problem $-\nabla \omega \nabla u=f$ with a scalar diffusion coefficient $\omega$ in $\Omega \subset \mathbb{R}^{2}$. Our estimates involve only very weak information about $\omega$, and the results are applicable for a large class of diffusion coefficients. Namely, we require $\omega$ to be in the Muckenhoupt class $A_{1}(\Omega)$, a function class well-studied in harmonic analysis.

The presented multilevel norm equivalencies are a main step towards the realization of an optimal and robust multilevel preconditioner for scalar diffusion problems.
\end{abstract}

\section{INTRODUCTION}

The solution of large sparse linear systems arising from the discretization of an elliptic partial differential equation (PDE) is an essential ingredient in many scientific computations. The ever growing demand for efficient solvers led to the development of multigrid methods in the 1970s [6, 7, 15, 16, 17] and multilevel preconditioning techniques in the late 1980s 24. Much research work was devoted to the question of optimal complexity, i.e., to show that the number of operations necessary to obtain the solution up to a prescribed accuracy is proportional to the number of unknowns of the linear system. Nevertheless, the convergence behavior of these classical schemes is still strongly dependent on the coefficients of the considered PDE. This is the so-called robustness problem of multilevel solvers, and it is one of the reasons which somewhat limit the applicability of classical multigrid methods and multilevel preconditioners in real world applications. Several extensions of multigrid methods e.g. via the use of more complicated smoothing schemes or through the use of operator-dependent or matrix-dependent transfer operators [1, 12, 26, 27] in the so-called black-box multigrid method have been proposed over the years to overcome the robustness problem. Currently the most successful approach is the algebraic multigrid (AMG) method [8, 9, 10, 11, 14, 19, 21] which

Received by the editor August 4, 2004 and, in revised form, August 3, 2006.

2000 Mathematics Subject Classification. Primary 65N55, 65F35; Secondary 65N30, 65F10.

Key words and phrases. Norm equivalency, multilevel method, preconditioning, robustness.

The authors were supported in part by the Sonderforschungsbereich 611 Singuläre Phänomene und Skalierung in Mathematischen Modellen sponsored by the Deutsche Forschungsgemeinschaft. 
further generalizes the black-box multigrid idea. AMG is a multiplicative multilevel scheme which employs information available from the system matrix (on the finest level) only. All other components, e.g. prolongations and coarse grid matrices, are constructed automatically in a purely algebraic fashion. The design and implementation of an AMG method is rather involved, and there is no satisfying theoretical foundation which proves the robustness and convergence of AMG, however, numerical experiments indicate the robustness of AMG. Hence, the development of a provably robust multiplicative multigrid solver remains an open problem even today. Note that there is no provably (or even practically) robust additive multilevel preconditioner for general elliptic second order PDEs.

In this paper we focus on the robustness issue for diffusion problems in two space dimensions involving a scalar diffusion coefficient $\omega: \mathbb{R}^{2} \rightarrow \mathbb{R}$; i.e., we consider the model problem

$$
-\nabla \omega \nabla u=f \text { in } \Omega \subset \mathbb{R}^{2} .
$$

We discretize our model problem using linear finite elements on a sequence of uniformly refined triangulations. Then, we can establish the equivalencies

$$
a(u, u) \asymp \sum_{j=0}^{J} 2^{2 j}\left\|u_{j}\right\|_{\omega}^{2} \quad \text { and } \quad a(u, u) \asymp \sum_{j=0}^{J} 2^{2 j}\left\|u_{j}\right\|_{j, \omega}^{2}
$$

for certain weighted norms $\|\cdot\|_{\omega}$ and $\|\cdot\|_{j, \omega}$ where the $u_{j}$ denote a decomposition of $u=\sum_{j} u_{j}$ obtained from orthogonal projections with respect to the weighted scalar product $\langle\cdot, \cdot\rangle_{\omega}$. These projections have also been studied in [2, 5, 25]. The constants of these equivalencies depend on the initial triangulation and involve some information about the variation of the coefficient function $\omega$ only. Namely, we require $\omega$ to be a weight from the Muckenhoupt class $A_{1}(\Omega)$. That is, we allow for highly oscillatory coefficient functions and do not require jumps of the coefficient function $\omega$ to be resolved on any level. This is in contrast to other articles concerned with the development of robust solvers [13, 18, 22,

The remainder of the paper is organized as follows: First, we introduce the notation and the employed norms in $\S 2$. Here, we also show the local equivalence of the considered weighted norms $\|\cdot\|_{\omega}$ and $\|\cdot\|_{j, \omega}$. Then, we present the main result of the paper in $\$ 3$. To this end, we establish two robust norm equivalencies for the considered model problem using a linear finite element discretization on a sequence of uniformly refined triangulations. We begin with the derivation of an optimal and robust upper bound for the bilinear form using a Bernstein-type inequality in trace norms, interpolation theory and a Hardy-inequality. To maintain the optimality of the estimate it is necessary to switch to trace norms and use an inequality of order $\frac{1}{2}$ due to the arbitrary discontinuities of the considered Muckenhoupt weights. Then, we establish a lower bound for the bilinear form using a local duality technique and a Hardy-inequality. Finally, we conclude with some remarks in $₫ 4$

\section{Prerequisites}

Let us introduce some notation which we will use throughout this paper. Our main interest is the development of robust multilevel solvers for diffusion problems

$$
-\nabla \omega \nabla u=f \text { in } \Omega \subset \mathbb{R}^{2}
$$


with the diffusion coefficient $\omega: \Omega \rightarrow \mathbb{R}$ being a scalar positive function. The associated bilinear form and energy norm are given by

$$
a(u, v):=\int_{\Omega} \omega \nabla u \nabla v \quad \text { and } \quad\|u\|_{a}:=\sqrt{a(u, u)},
$$

respectively. We consider a sequence of uniformly refined triangulations of $\Omega$

$$
\mathcal{T}_{0} \subset \mathcal{T}_{1} \subset \cdots \subset \mathcal{T}_{J}
$$

with the associated sequence of piecewise linear finite element spaces

$$
\mathcal{V}_{0} \subset \mathcal{V}_{1} \subset \cdots \subset \mathcal{V}_{J}
$$

Note that we employ linear finite elements $u \in \mathcal{V}_{j}$ only. Therefore $\nabla u$ is constant on each element $T \in \mathcal{T}_{j}$ and we have

$$
\int_{T} \omega \nabla u \nabla v=\left.(\nabla u \nabla v)\right|_{T} \int_{T} \omega \text { for all } u, v \in \mathcal{V}_{j}
$$

Let us now introduce the discrete weight

$$
\omega_{T}:=\frac{1}{\mu(T)} \int_{T} \omega \text { for } T \in \mathcal{T}_{j} \text { and any } j=0, \ldots, J,
$$

where $\mu$ denotes the Lebesgue measure. With these discrete weights and (2.3) we obtain

$$
a(u, v)=\int_{\Omega} \omega \nabla u \nabla v=\sum_{T \in \mathcal{T}_{j}} \omega_{T} \int_{T} \nabla u \nabla v \quad \text { for } u, v \in \mathcal{V}_{j}
$$

Note that due to the nestedness of the triangulations $\mathcal{T}_{j} \subset \mathcal{T}_{k}$ with $j \leq k \leq J$, and $j=0, \ldots, J$, we have

$$
\omega_{\hat{T}}=\frac{1}{\mu(\hat{T})} \int_{\hat{T}} \omega=\frac{1}{\mu(\hat{T})} \sum_{\substack{T \subset \hat{T} \\ T \in \mathcal{T}_{J}}} \mu(T) \omega_{T}
$$

for any element $\hat{T} \in \mathcal{T}_{j}$. On each level $j=0, \ldots, J$ we define the scalar products

$$
\langle u, v\rangle_{j, \omega}:=\sum_{\hat{T} \in \mathcal{T}_{j}} \omega_{\hat{T}} \int_{\hat{T}} u v, \quad \text { for } u, v \in \mathcal{V}_{j}
$$

and associated weighted $L^{2}$-norms

$$
\|u\|_{j, \omega}^{2}:=\langle u, u\rangle_{j, \omega}=\sum_{\hat{T} \in \mathcal{T}_{j}} \omega_{\hat{T}}\|u\|_{\hat{T}}^{2}=\sum_{\hat{T} \in \mathcal{T}_{j}} \omega_{\hat{T}} \int_{\hat{T}}|u|^{2}
$$

using the discrete weights (2.5). On the finest level $J$ we furthermore introduce the shorthand notation

$$
\langle\cdot, \cdot\rangle_{\omega}:=\langle\cdot, \cdot\rangle_{J, \omega} \quad \text { and } \quad\|\cdot\|_{\omega}:=\|\cdot\|_{J, \omega} .
$$

In this paper, we consider weights $\omega$, i.e., locally integrable positive functions, which belong to the Muckenhoupt class $A_{1}(\Omega)$ [20] only.

Definition 2.1 (Muckenhoupt class $A_{1}(\Omega)$ ). A weight $\omega: \Omega \rightarrow \mathbb{R}$ is in the class $A_{1}(\Omega)$ if and only if there is a constant $c(\omega)$ such that the inequality

$$
\frac{\left\|\omega^{-1}\right\|_{L_{\infty}(\Omega)}}{\mu(B)} \int_{B} \omega \leq c(\omega)<\infty
$$


holds for all balls $B \subset \Omega$. The smallest such constant is referred to as $|\omega|_{A_{1}(\Omega)}:=$ $\min c(\omega)$. As a consequence, the inequality

$$
\omega_{B}=\frac{1}{\mu(B)} \int_{B} \omega \leq|\omega|_{A_{1}(\Omega)} \frac{1}{\mu(F)} \int_{F} \omega=|\omega|_{A_{1}(\Omega)} \omega_{F}
$$

holds for all $\omega \in A_{1}(\Omega)$ and all balls $B \subset \Omega$ and all subsets $F \subset B$.

In the following, we employ property (2.8) in a slightly modified form only. Namely, we use triangles $T \in \mathcal{T}_{j}$ on any level $j=0, \ldots, J$ instead of balls $B$ and the union of triangles $\hat{T} \in \mathcal{T}_{J}$ on the finest level instead of the subsets $F$. Hence, our results hold for a slightly larger class of functions, but it is sufficient to require $\omega \in A_{1}(\Omega)$.

For such weights, we show a local Bernstein-type inequality in Lemma 2.2 which will be crucial for the proof of the local norm equivalence of the norms $\|\cdot\|_{j, \omega}$ and $\|\cdot\|_{\omega}=\|\cdot\|_{J, \omega}$ in Theorem 2.3. Note that this theorem is an essential ingredient in the later proof of our norm equivalence. In fact, we will show an upper bound for the bilinear form $a(u, u)$ with the level-dependent $\|\cdot\|_{j, \omega}$ norms directly in section 3.1. The lower bound, however, we establish for the $\|\cdot\|_{\omega}$ norm in section 3.2. Hence, to attain a complete norm equivalence for either norm we need to employ Theorem 2.3.

Lemma 2.2. Let $\omega$ be in the Muckenhoupt class $A_{1}(\Omega)$. Consider a sequence of uniformly refined triangulations $\mathcal{T}_{j}$ and the associated sequence of nested spaces $\mathcal{V}_{j}$ of linear finite elements. Then there holds for the norm $\|\cdot\|_{J, \omega}=\|\cdot\|_{\omega}$ defined in (2.7) the local Bernstein-type inequality

$$
\|\nabla v\|_{\omega, T} \leq C_{B}|\omega|_{A_{1}(\Omega)}^{\frac{1}{2}}(\operatorname{diam}(T))^{-1}\|v\|_{\omega, T}, \text { with } C_{B}:=\frac{3}{2} \sqrt{2} C_{0}
$$

for all $v \in \mathcal{V}_{j}$ and any $j=0, \ldots, J$. Here $\|\cdot\|_{\omega, T}$ denotes the restriction of the norm $\|\cdot\|_{\omega}$ to $T \in \mathcal{T}_{j}$, and the constant $C_{0}$ is only dependent on the initial triangulation $\mathcal{T}_{0}$, i.e.,

$$
C_{0}:=\max _{\hat{T} \in \mathcal{T}_{0}} \operatorname{diam}(\hat{T})\left\|H_{\hat{T}}^{-1}\right\|
$$

where $H_{S}$ denotes the mapping from $S$ to the reference triangle $T_{\text {ref }}$ with vertices $(0,0),(0,1),(1,0)$.

Proof. Let $H_{T}$ denote the mapping from $T \in \mathcal{T}_{j}$ for any $j=0, \ldots, J$ to the reference triangle $T_{\text {ref }}$, such that for $x \in T$ we have $H_{T}(x)=\xi \in T_{\text {ref }}$. Since we consider linear elements only, we have the representation

$$
v(x)=q \circ H_{T}(x)=q(\xi)=(g, \xi)+e
$$

for any $v \in \mathcal{V}_{j}$ on $T$ with $g=\nabla_{\xi} q$. By the mean value theorem, there exists $x_{\hat{T}} \in \hat{T} \in \mathcal{T}_{J}, \hat{T} \subset T$ with $\int_{\hat{T}}|v|^{2}=\mu(\hat{T}) v\left(x_{\hat{T}}\right)^{2}$. Let $\xi_{\hat{T}} \in T_{\text {ref }}$ denote the reference image of $x_{\hat{T}}$, i.e., $H_{T}\left(x_{\hat{T}}\right)=\xi_{\hat{T}}$. By definition (2.6) and the representation (2.11) we obtain the identity

$$
\|v\|_{\omega, T}^{2}=\sum_{\substack{\hat{T} \subset T \\ \hat{T} \in \mathcal{T}_{J}}} \omega_{\hat{T}} \int_{\hat{T}}|v|^{2}=\sum_{\substack{\hat{T} \subset T \\ \hat{T} \in \mathcal{T}_{J}}} \omega_{\hat{T}} \mu(\hat{T})\left|\left(g, \xi_{\hat{T}}\right)+e\right|^{2} .
$$

Furthermore, with $C_{0}$ given in (2.10) there holds the pointwise inequality

$$
|\nabla v(x)|^{2} \leq C_{0}^{2}(\operatorname{diam}(T))^{-2}\|g\|^{2} \quad \text { for all } x \in T
$$


where $\|\cdot\|$ denotes the Euclidian norm in $\mathbb{R}^{2}$. Thus, we obtain

$$
\|\nabla v\|_{\omega, T}^{2}=\sum_{\substack{\hat{T} \subset T \\ \hat{T} \in \mathcal{T}_{J}}} \omega_{\hat{T}} \int_{\hat{T}}|\nabla v|^{2} \leq C_{0}^{2}(\operatorname{diam}(T))^{-2}\|g\|^{2} \sum_{\substack{\hat{T} \subset T \\ \hat{T} \in \mathcal{T}_{J}}} \omega_{\hat{T}} \mu(\hat{T}) .
$$

Hence, it suffices to prove the local inequality

$$
\|g\|^{2} \sum_{\substack{\hat{T} \in \mathcal{T}_{J} \\ \hat{T} \subset T}} \omega_{\hat{T}} \mu(\hat{T})=\|g\|^{2} \int_{T} \omega \leq c \sum_{\substack{\hat{T} \in \mathcal{T}_{J} \\ \hat{T} \subset T}} \omega_{\hat{T}} \mu(\hat{T})\left|\left(g, \xi_{\hat{T}}\right)+e\right|^{2}=c\|v\|_{\omega, T}^{2}
$$

for any $T \in \mathcal{T}_{j}$ and some $c>0$ to show the assertion (2.9). To this end, we first consider the case $e=0$ and set $b:=\frac{g}{\|g\|}$. Then inequality (2.12) simplifies to

$$
\int_{T} \omega \leq c \sum_{\substack{\hat{T} \subset T \\ \hat{T} \in \mathcal{T}_{J}}} \omega_{\hat{T}} \mu(\hat{T})\left|\left(b, \xi_{\hat{T}}\right)\right|^{2}
$$

for all $b$ with $\|b\|=1$. Then we define for any $\rho \in(0,1)$ the subregion $S_{\rho} \subset T \in \mathcal{T}_{j}$ by

$$
S_{\rho}:=\left\{x \in T: H_{T}(x)=\xi \in S_{\text {ref }, \rho}\right\}, \quad S_{\text {ref }, \rho}:=\left\{\xi \in T_{\text {ref }}:|(b, \xi)| \geq \rho\right\} .
$$

Suppose that

$$
\mu\left(S_{\rho}\right)=\alpha \mu(T)
$$

then by inequality (2.8) we have

$$
\left.\sum_{\substack{\hat{T} \subset T \\ \hat{T} \in \mathcal{T}_{J}}} \omega_{\hat{T}} \mu(\hat{T})||\left(b, \xi_{\hat{T}}\right)\right|^{2} \geq \rho^{2} \sum_{\substack{x_{\hat{T}} \in S_{\rho} \\ \hat{T} \in \mathcal{T}_{J}}} \omega_{\hat{T}} \mu(\hat{T})=\rho^{2} \int_{S_{\rho}} \omega \geq \frac{\alpha \rho^{2}}{|\omega|_{A_{1}(\Omega)}} \int_{T} \omega
$$

and assertion (2.13) holds with $c=\frac{|\omega|_{A_{1}(\Omega)}}{\alpha \rho^{2}}$.

It remains to show (2.14). Note that the boundary of the subset $S_{\text {ref, } \rho}$ is given by sections of the line $|(b, \xi)|=\rho$ and the boundary of $T_{\text {ref }}$. The line $|(b, \xi)|=\rho$ is orthogonal to the vector $b$ and has $\xi_{0}:=\rho \cdot b$ as its nearest point to the origin with distance $\rho$. Varying the angle of $b$ with the axis $\xi_{2}=0$ one easily sees that for fixed $\rho>0$ the minimum for the area $\mu\left(S_{\mathrm{ref}, \rho}\right)$ is achieved when $b$ is parallel to one of the axes $\xi_{1}=\rho$ or $\xi_{2}=\rho$. In this case we have $\mu\left(S_{\text {ref }, \rho}\right)=(1-\rho) \mu\left(T_{\text {ref }}\right)$ and hence $\mu(S)=(1-\rho) \mu(T)$ so that (2.14) holds with $\alpha=1-\rho$ and $c=\frac{|\omega|_{A_{1}(\Omega)}}{(1-\alpha)^{2} \alpha}$. Therefore, we obtain the best possible constant $c=\frac{27}{4}|\omega|_{A_{1}(\Omega)}$ by a choice of $\alpha=\frac{1}{3}$.

The general case can be reduced to this special case by considering $\tilde{v}(x):=$ $v(x)-e$ instead of $v(x)$ and observing that $\|\nabla \tilde{v}\|_{\omega, T}=\|\nabla v\|_{\omega, T}$. Since $v(x)$ is linear, we can assume without loss of generality that $e=\min _{x \in T}|v(x)|$ and we can estimate

$$
\int_{T}|\tilde{v}(x)|^{2} d x \leq 2 \int_{T}\left(|v(x)|^{2}+|e|^{2}\right) d x \leq 4 \int_{T}|v(x)|^{2} d x .
$$

With the help of Lemma 2.2 we now obtain the local equivalence of the weighted norms $\|\cdot\|_{j, \omega}$ and $\|\cdot\|_{\omega}$ for $v \in \mathcal{V}_{j}$ on all levels $j=0, \ldots, J$. 
Theorem 2.3. Let $\omega$ be in the Muckenhoupt class $A_{1}(\Omega)$ and consider a sequence of uniformly refined triangulations $\mathcal{T}_{j}$ and the associated sequence of nested spaces $\mathcal{V}_{j}$ of linear finite elements. Then there holds for the norms $\|\cdot\|_{j, \omega}$ and $\|\cdot\|_{J, \omega}=\|\cdot\|_{\omega}$ defined in (2.6) for $v \in \mathcal{V}_{j}$ and $T \in \mathcal{T}_{j}$

$$
\left(1+C_{B}|\omega|_{A_{1}(\Omega)}^{\frac{1}{2}}\right)^{-1}\|v\|_{j, \omega, T} \leq\|v\|_{\omega, T} \leq\left(1+6 C_{0}\right)\|v\|_{j, \omega, T}
$$

with the constant $C_{0}$ only depending on the initial triangulation $\mathcal{T}_{0}$.

Proof. For $v \in \mathcal{V}_{j}$ there holds by definition

$$
\left|\|v\|_{j, \omega, T}-\|v\|_{\omega, T}\right|=\left|\sqrt{\omega_{T} \int_{T}|v|^{2}}-\sqrt{\sum_{\substack{\hat{T} \in \mathcal{T} J \\ \hat{T} \subset T}} \omega_{\hat{T}} \int_{\hat{T}}|v|^{2}}\right| .
$$

Now by the mean value theorem there exist $\xi_{T} \in T \in \mathcal{T}_{j}$ and $\xi_{\hat{T}} \in \hat{T} \in \mathcal{T}_{J}$ with

$$
\frac{1}{\mu(T)} \int_{T}|v|^{2}=\left|v\left(\xi_{T}\right)\right|^{2} \quad \text { and } \quad \frac{1}{\mu(\hat{T})} \int_{\hat{T}}|v|^{2}=\left|v\left(\xi_{\hat{T}}\right)\right|^{2},
$$

respectively. Plugging this into (2.16) and using (2.5) we obtain

$$
\begin{aligned}
\left|\|v\|_{j, \omega, T}-\|v\|_{\omega, T}\right| & =\left|\sqrt{\sum_{\substack{\hat{T} \in \mathcal{T}_{J} \\
\hat{T} \subset T}} \omega_{\hat{T}} \mu(\hat{T})\left|v\left(\xi_{T}\right)\right|^{2}}-\sqrt{\sum_{\substack{\hat{T} \in \mathcal{T}_{J} \\
\hat{T} \subset T}} \omega_{\hat{T}} \mu(\hat{T})\left|v\left(\xi_{\hat{T}}\right)\right|^{2}}\right| \\
\leq & \sqrt{\sum_{\substack{\hat{T} \in \mathcal{T}_{J} \\
\hat{T} \subset T}} \omega_{\hat{T}} \mu(\hat{T})\left|v\left(\xi_{T}\right)-v\left(\xi_{\hat{T}}\right)\right|^{2}} .
\end{aligned}
$$

With the help of the estimate

$$
\left|v\left(\xi_{\hat{T}}\right)-v\left(\xi_{T}\right)\right|^{2} \leq\|\nabla v\|^{2}\left\|\xi_{\hat{T}}-\xi_{T}\right\|^{2} \leq\|\nabla v\|^{2}(\operatorname{diam}(T))^{2},
$$

the inequality

$$
\left|\|v\|_{j, \omega, T}-\|v\|_{\omega, T}\right| \leq \operatorname{diam}(T)\|\nabla v\|_{\omega, T}
$$

follows. Combining this with the local Bernstein-type inequality of Lemma 2.2, we arrive at the asserted left-hand estimate

$$
\|v\|_{j, \omega, T} \leq \operatorname{diam}(T)\|\nabla v\|_{\omega, T}+\|v\|_{\omega, T} \leq\left(1+C_{B}|\omega|_{A_{1}(\Omega)}^{\frac{1}{2}}\right)\|v\|_{\omega, T} .
$$

Denoting the vertices of $T$ by $x_{T, i}$ with $i=1,2,3$ and $v\left(x_{T, i}\right)=w_{T, i}$ there holds the representation $g=\left(w_{T, 2}-w_{T, 1}, w_{T, 3}-w_{T, 1}\right)^{t}$. With the help of the equivalence, see e.g. [3,

$$
\frac{1}{\mu(T)} \int_{T}|v|^{2}=\frac{w_{T, 3}^{2}+w_{T, 2}^{2}+w_{T, 1}^{2}+\left(w_{T, 3}+w_{T, 2}+w_{T, 1}\right)^{2}}{12}
$$

and the inequality

$$
\left(w_{T, 2}-w_{T, 1}\right)^{2}+\left(w_{T, 3}-w_{T, 1}\right)^{2} \leq 3\left(w_{T, 3}^{2}+w_{T, 2}^{2}+w_{T, 1}^{2}+\left(w_{T, 3}+w_{T, 2}+w_{T, 1}\right)^{2}\right)
$$

we obtain

$$
\operatorname{diam}(T)\|\nabla v\|_{\omega, T} \leq 6 C_{0}\|v\|_{j, \omega, T}
$$

which proves the asserted right-hand inequality. 


\section{ROBUST NORM EQUIVALENCIES}

The aim of this section is to establish two robust norm equivalencies for our model problem (2.1). Namely, we are interested in the equivalence

$$
a(u, u) \asymp \sum_{j=1}^{J} 2^{2 j}\left\|u_{j}\right\|_{j, \omega}^{2}+a\left(u_{0}, u_{0}\right)
$$

and the equivalence

$$
a(u, u) \asymp \sum_{j=1}^{J} 2^{2 j}\left\|u_{j}\right\|_{\omega}^{2}+a\left(u_{0}, u_{0}\right)
$$

where the decomposition $u_{j}$ is obtained from orthogonal projections with respect to the weighted scalar product (2.7) on the finest level.

3.1. Upper bounds for the bilinear form. We begin with the derivation of an upper bound for the bilinear form $a(u, u)$, which is robust and optimal, i.e., it does not involve the factor $J$ and weak information about $\omega$ only. We obtain our estimate in three steps: First, we introduce a sequence of projection operators $Q_{j}^{\omega}$ based on the weighted scalar product $\langle\cdot, \cdot\rangle_{\omega}=\langle\cdot, \cdot\rangle_{J, \omega}$ on the finest level $J$. Then, we apply a Bernstein-type estimate to the bilinear form $a\left(\sum_{j} u_{j}, \sum_{l} u_{l}\right)$ using the decomposition $u=\sum_{j} u_{j}$ of (3.9). Here, the challenge is to bound the mixed terms in the arising double sum. One approach to this issue is the use of a strengthened Cauchy-Schwarz inequality, or one applies interpolation theory to $a(u, u)$ and works with a Bernstein-type inequality of second order and a Hardytype inequality for the arising sum. However, it seems that these approaches do not work if the diffusion coefficient $\omega$ is discontinuous. Therefore, we need to consider a second sequence of projection operators $Q_{j}^{a}$ based on auxiliary bilinear forms $a_{j}(\cdot, \cdot)$ which are defined in a level-dependent fashion. With the help of the two projections $Q_{j}^{\omega}$ and $Q_{j}^{a}$, we establish a hybrid Bernstein-type inequality involving both projections. Furthermore, we use averages of the weight $\omega$ and a Bernsteintype inequality in trace norms which correspond to inequalities of order $\frac{1}{2}$. Then, we can use a Hardy-type inequality to deal with the arising sums. Finally, we derive a robust and optimal upper bound of $a(\cdot, \cdot)$ in Theorem 3.3 using only the projections $Q_{j}^{\omega}$ via a Hardy inequality.

A tool that is used in the proof of Theorem 3.3 is the following Bernstein-type inequality of broken order for the weighted trace norms

$$
\|u\|_{\frac{1}{2}, j, \omega}:=\left(\sum_{T \in \mathcal{T}_{j}} \omega_{T} \int_{\partial T}|u|^{2}\right)^{\frac{1}{2}} .
$$

Lemma 3.1. Let $\omega$ be a locally integrable positive function and consider a sequence of uniformly refined triangulations $\mathcal{T}_{j}$ and the associated sequence of nested spaces $\mathcal{V}_{j}$ of linear finite elements. For elements $v \in \mathcal{V}_{j}$ there holds

$$
\|v\|_{a} \leq \sqrt{3 C_{2} C_{1}} 2^{\frac{j}{2}}\|v\|_{\frac{1}{2}, j, \omega}
$$

where the constants $C_{1}:=\max _{T \in \mathcal{T}_{0}} \operatorname{diam}(T)$ and $C_{2}:=\max _{\hat{T} \in \mathcal{T}_{0}} \frac{\operatorname{diam}(\hat{T})}{\sqrt{\mu(\hat{T})}}$ depend on the initial triangulation $\mathcal{T}_{0}$ only. 
Proof. Keeping in mind that we employ linear finite elements only, we have that $\nabla v$ is constant on $T \in \mathcal{T}_{j}$. Integration by parts on each $T \in \mathcal{T}_{j}$ then yields

$$
a(v, v)=\sum_{T \in \mathcal{T}_{j}} \omega_{T} \int_{T} \nabla v \nabla v=\sum_{T \in \mathcal{T}_{j}} \omega_{T} \int_{\partial T} v \nabla v \cdot n_{\partial T}
$$

Applying the Cauchy-Schwarz inequality gives

$$
a(v, v) \leq\left(\sum_{T \in \mathcal{T}_{j}} \omega_{T} \int_{\partial T}|v|^{2}\right)^{\frac{1}{2}}\left(\sum_{T \in \mathcal{T}_{j}} \omega_{T} \int_{\partial T}\|\nabla v\|^{2}\right)^{\frac{1}{2}} .
$$

Here, the first sum represents the semi-norm $\|v\|_{\frac{1}{2}, j, \omega}$, whereas each term in the second sum can be bounded using the local Bernstein-type inequality

$$
\int_{\partial T}\|\nabla v\|^{2} \leq 3 \operatorname{diam}(T)\|\nabla v\|^{2} \leq 3 C_{2} \mu(T)^{\frac{1}{2}}\|\nabla v\|^{2} \leq 3 C_{2} C_{1} 2^{j} \int_{T}\|\nabla v\|^{2} .
$$

Hence, after multiplication with $\omega_{T}$ and summation with respect to $T \in \mathcal{T}_{j}$, we obtain the overall estimate

$$
a(v, v)=\sum_{T \in \mathcal{T}_{j}} \omega_{T} \int_{T} \nabla v \nabla v \leq \sqrt{3 C_{2} C_{1}} 2^{\frac{j}{2}}\|v\|_{\frac{1}{2}, j, \omega} \sqrt{a(v, v)}
$$

and the assertion follows after division by $\sqrt{a(v, v)}$.

Let us now introduce some additional notation. We start with the definition of a sequence of weighted projections $Q_{j}^{\omega}: \mathcal{V}_{J} \rightarrow \mathcal{V}_{j}$ by the relation

$$
\left\langle Q_{j}^{\omega} u, v\right\rangle_{\omega}:=\langle u, v\rangle_{\omega}
$$

for all $u \in \mathcal{V}_{J}$ and $v \in \mathcal{V}_{j}$. Furthermore, we define auxiliary level-dependent bilinear forms $a_{j}: \mathcal{V}_{J} \times \mathcal{V}_{j} \rightarrow \mathbb{R}$ by

$$
a_{j}(u, v):=\sum_{T \in \mathcal{T}_{j}} \omega_{T} \int_{T} \nabla u \nabla v=\sum_{T \in \mathcal{T}_{j}} \frac{1}{\mu(T)} \sum_{\substack{\hat{T} \in \mathcal{T}_{J} \\ \hat{T} \subset T}} \mu(\hat{T}) \omega_{\hat{T}} \int_{T} \nabla u \nabla v .
$$

Note that for $u, v \in \mathcal{V}_{j}$ we have $a(u, v)=a_{j}(u, v)$ and with (2.5) it follows that for $j \leq k$

$$
\begin{aligned}
a_{k}(u, v) & =\sum_{T \in \mathcal{T}_{k}} \omega_{T} \int_{T} \nabla u \nabla v=\left.\sum_{T \in \mathcal{T}_{k}} \omega_{T} \mu(T)(\nabla u \nabla v)\right|_{T} \\
& =\left.\sum_{\hat{T} \in \mathcal{T}_{j}} \sum_{\substack{T \in \mathcal{T}_{k} \\
T \subset T}} \omega_{T} \mu(T)(\nabla u \nabla v)\right|_{\hat{T}}=\left.\sum_{\hat{T} \in \mathcal{T}_{j}} \omega_{\hat{T}}(\nabla u \nabla v)\right|_{\hat{T}} \\
& =\sum_{T \in \mathcal{T}_{j}} \omega_{T} \int_{T} \nabla u \nabla v=a_{j}(u, v)
\end{aligned}
$$

since $\left.(\nabla u \nabla v)\right|_{\hat{T}}=\left.(\nabla u \nabla v)\right|_{T}$ for all $T \subset \hat{T} \in \mathcal{T}_{j}$. With the help of these auxiliary bilinear forms and the direct splitting $\mathcal{V}_{j}=V_{j-1} \oplus \mathcal{W}_{j}$ we define the generalized projection operators $P_{j}^{a}: \mathcal{V}_{J} \rightarrow \mathcal{V}_{j}$ and $Q_{j}^{a}: \mathcal{V}_{J} \rightarrow \mathcal{V}_{j}$ by

$$
a_{j}\left(P_{j}^{a} u, v\right)=a\left(P_{j}^{a} u, v\right)=\left\{\begin{array}{ll}
0 & v \in \mathcal{V}_{j-1} \\
a_{j}(u, v) & v \in \mathcal{W}_{j}
\end{array}, \quad \text { and } Q_{j}^{a}:=\sum_{k=1}^{j} P_{k}^{a}\right.
$$

for all $u \in \mathcal{V}_{J}$. Consider $v \in \mathcal{V}_{j-1}$; due to (3.4) we obtain

$$
a_{j}\left(Q_{j}^{a} u-Q_{j-1}^{a} u, v\right)=a_{j}\left(Q_{j}^{a} u, v\right)-a_{j-1}\left(Q_{j-1}^{a} u, v\right)
$$


and with (3.5) this yields

$$
a_{j}\left(Q_{j}^{a} u-Q_{j-1}^{a} u, v\right)=0 \quad \text { for all } v \in \mathcal{V}_{j-1} .
$$

Hence, the decomposition

$$
v_{j}=v_{j}(u):=Q_{j}^{a} u-Q_{j-1}^{a} u, \quad v_{0}:=Q_{0}^{a} u
$$

associated with any $u \in \mathcal{V}_{J}$ has the property

$$
a\left(v_{j}, v_{k}\right)=0 \quad \text { for all } v \in \mathcal{V}_{j} \text { with } j<k
$$

due to (3.6). Since $u=Q_{J}^{a} u=\sum_{j=0}^{J} v_{j}$ this yields the equivalence

$$
a(u, u)=\sum_{j, k=0}^{J} a\left(v_{j}, v_{k}\right)=\sum_{j=0}^{J} a\left(v_{j}, v_{j}\right)=\sum_{j=0}^{J}\left\|v_{j}\right\|_{a}^{2}
$$

for any $u \in \mathcal{V}_{J}$ on the finest level.

Finally, we introduce the sequence

$$
u_{k}=u_{k}(u):=Q_{k}^{\omega} u-Q_{k-1}^{\omega} u, \quad u_{0}:=Q_{0}^{\omega} u, \quad \text { for } u \in \mathcal{V}_{J}
$$

based on the projections $Q_{k}^{\omega}$.

In the following we consider $u \in \mathcal{V}_{J}$ on the finest level $J$ and its associated decompositions $v_{j}$ from (3.7) and $u_{j}$ from (3.9). We obtain an upper bound for $\left\|v_{j}\right\|_{a}$ in terms of $\left\|u_{k}\right\|_{k, \omega}$ for $j \leq k$; i.e., we establish a hybrid Bernstein-type estimate of order $\frac{1}{2}$ of $a\left(v_{j}, v_{j}\right)$ in terms of $\|u\|_{\frac{1}{2}, k, \omega}$ for $j \leq k$.

Lemma 3.2. Let $\omega$ be a locally integrable positive function and consider a sequence of uniformly refined triangulations $\mathcal{T}_{j}$ and the associated sequence of nested spaces $\mathcal{V}_{j}$ of linear finite elements. Let $u \in \mathcal{V}_{J}, v_{j} \in \mathcal{V}_{j}$ and $u_{k} \in \mathcal{V}_{k}$ for $k \geq j$ be defined as in (3.7) and (3.9), respectively. Then there holds the estimate

$$
a\left(v_{j}, v_{j}\right) \leq 3 C_{2} C_{1} 2^{j}\left(\sum_{k=j}^{J}\left\|u_{k}\right\|_{\frac{1}{2}, j, \omega}\right)^{2}
$$

with constants $C_{1}, C_{2}$ from Lemma 3.1 .

Proof. Recall the definition (3.7) of $v_{j}$. Due to (3.5), we have $a\left(v_{j}, w\right)=0$ for all $w \in \mathcal{V}_{j-1}$. Hence, with the choice $w=Q_{j-1}^{\omega} u$ we obtain

$$
\begin{aligned}
a\left(v_{j}, v_{j}\right) & =a_{j}\left(v_{j}, v_{j}\right)=a_{j}\left(v_{j}, u\right)=a_{j}\left(v_{j}, u-Q_{j-1}^{\omega} u\right) \\
& =\sum_{T \in \mathcal{T}_{j}} \omega_{T} \int_{T} \nabla v_{j} \nabla\left(u-Q_{j-1}^{\omega} u\right) .
\end{aligned}
$$

From the definition (3.9) we obtain the identity $u-Q_{j-1}^{\omega} u=\sum_{k=j}^{J} Q_{k}^{\omega} u-Q_{k-1}^{\omega} u=$ $\sum_{k=j}^{J} u_{k}$ and we can establish the equivalence

$$
a\left(v_{j}, v_{j}\right)=\sum_{T \in \mathcal{T}_{j}} \omega_{T} \sum_{k=j}^{J} \int_{T} \nabla v_{j} \nabla u_{k} .
$$

Integration by parts on each $T \in \mathcal{T}_{j}$ yields

$$
a\left(v_{j}, v_{j}\right)=\sum_{k=j}^{J} \sum_{T \in \mathcal{T}_{j}} \omega_{T} \int_{\partial T} u_{k} \nabla v_{j} \cdot n_{\partial T},
$$


and we obtain

$$
\sum_{T \in \mathcal{T}_{j}} \omega_{T} \int_{\partial T} u_{k} \nabla v_{j} \cdot n_{\partial T} \leq\left(\sum_{T \in \mathcal{T}_{j}} \omega_{T} \int_{\partial T}\left|u_{k}\right|^{2}\right)^{\frac{1}{2}}\left(\sum_{T \in \mathcal{T}_{j}} \omega_{T} \int_{\partial T}\left\|\nabla v_{j}\right\|^{2}\right)^{\frac{1}{2}}
$$

as in the proof of Lemma 3.1. The second factor can be estimated in the same way by

$$
\left(\sum_{T \in \mathcal{T}_{j}} \omega_{T} \int_{\partial T}\left\|\nabla v_{j}\right\|^{2}\right)^{\frac{1}{2}} \leq \sqrt{3 C_{2} C_{1}} 2^{j / 2} \sqrt{a\left(v_{j}, v_{j}\right)}
$$

whereas the first factor is by definition (3.1) the norm $\left\|u_{k}\right\|_{\frac{1}{2}, j, \omega}$. This yields the assertion.

Note that (3.10) is an inequality of weak-type. Such inequalities are for instance used for the estimates of the K-functional between Sobolev spaces.

With the help of this lemma we can now show the main result of this subsection, the robust and optimal upper bound for the bilinear form (2.2).

Theorem 3.3. Let $\omega$ be in the Muckenhoupt class $A_{1}(\Omega)$ and consider a sequence of uniformly refined triangulations $\mathcal{T}_{j}$ and the associated sequence of nested spaces $\mathcal{V}_{j}$ of linear finite elements. Then there holds the upper bound

$$
a(u, u) \leq C_{U}^{2}|\omega|_{A_{1}(\Omega)} \sum_{j=0}^{J} 2^{2 j}\left\|u_{j}\right\|_{j, \omega}^{2}, \text { with } C_{U}:=C_{2} C_{1}(2+\sqrt{2})
$$

for $u \in \mathcal{V}_{J}$ and its associated decomposition $u_{j}$ from (3.9), $\|\cdot\|_{j, \omega}$ is given in (2.6), and the constants $C_{1}$ and $C_{2}$ are stated in Lemma 3.1. With respect to the $\|\cdot\|_{\omega}$ norm on the finest level $J$ there holds the estimate

$$
a(u, u) \leq\left(1+C_{B}|\omega|_{A_{1}(\Omega)}^{\frac{1}{2}}\right)^{2} C_{U}^{2}|\omega|_{A_{1}(\Omega)} \sum_{j=0}^{J} 2^{2 j}\left\|u_{j}\right\|_{\omega}^{2} .
$$

Proof. Consider a fixed $T \in \mathcal{T}_{j}$ and $U \in \mathcal{T}_{k}$ with $k \geq j$ and $U \subset T$. Applying property (2.8) of $\omega$ to $F=U$ and $B=T$ we obtain

$$
\sum_{T \in \mathcal{T}_{j}} \omega_{T} \int_{\partial T}\left|u_{k}\right|^{2} \leq \sum_{T \in \mathcal{T}_{j}} \sum_{\substack{U \in \mathcal{T}_{k} \\ U \subset T}} \omega_{T} \int_{\partial U}\left|u_{k}\right|^{2} \leq|\omega|_{A_{1}(\Omega)} \sum_{U \in \mathcal{T}_{k}} \omega_{U} \int_{\partial U}\left|u_{k}\right|^{2} .
$$

With the help of Lemma 3.2 this yields

$$
\left\|v_{j}\right\|_{a}^{2} \leq 3 C_{2} C_{1}|\omega|_{A_{1}(\Omega)}\left(\sum_{k=j}^{J}\left\|u_{k}\right\|_{\frac{1}{2}, k, \omega}\right)^{2},
$$

and with Lemma 3.1 we can estimate

$$
a(u, u)=\sum_{j=0}^{J}\left\|v_{j}\right\|_{a}^{2} \leq\left(3 C_{2} C_{1}\right)^{2}|\omega|_{A_{1}(\Omega)} \sum_{j=0}^{J} 2^{j}\left(\sum_{k=j}^{J} 2^{k / 2}\left\|u_{k}\right\|_{k, \omega}\right)^{2} .
$$

Using the Hardy inequality

$$
\left(\sum_{j=0}^{J} b^{j}\left(\sum_{k=j}^{J} a_{k}\right)^{2}\right)^{\frac{1}{2}} \leq \frac{1}{1-1 / \sqrt{b}}\left(\sum_{k=0}^{J} b^{k} a_{k}^{2}\right)^{\frac{1}{2}}
$$

with $a_{k}:=2^{k / 2}\left\|u_{k}\right\|_{k, \omega}$, and $b=2$ we establish the asserted optimal and robust upper bound (3.11). 
Applying Theorem 2.3. we obtain the corresponding upper bound (3.12) of $a(u, u)$ for the weighted norm $\|\cdot\|_{\omega}$ on the finest level $J$.

3.2. Lower bounds for the bilinear form. The next step in our search for robust norm equivalencies is the derivation of optimal and robust lower bounds for the bilinear form $a(\cdot, \cdot)$ in terms of the norms $\|\cdot\|_{j, \omega}$ and $\|\cdot\|_{\omega}$; i.e., we are looking for the Jackson-type inequalities

$$
\sum_{j=1}^{J} 2^{2 j}\left\|u_{j}\right\|_{j, \omega}^{2}+a\left(u_{0}, u_{0}\right) \leq C a(u, u) \quad \text { and } \quad \sum_{j=1}^{J} 2^{2 j}\left\|u_{j}\right\|_{\omega}^{2}+a\left(u_{0}, u_{0}\right) \leq C a(u, u) .
$$

To this end, we split the sum $\sum_{j=1}^{J} 2^{2 j}\left\|u_{j}\right\|_{\omega}^{2}$ into two parts, $\sum_{j=1}^{j_{0}-1} 2^{2 j}\left\|u_{j}\right\|_{\omega}^{2}$ and $\sum_{j=j_{0}}^{J} 2^{2 j}\left\|u_{j}\right\|_{\omega}^{2}$. Here, the parameter $j_{0}$ is independent of $J$ and $j_{0}$ depends on the considered weight function $\omega$ and the initial triangulation $\mathcal{T}_{0}$ only. The constants of the lower bound will explicitly involve $j_{0}$. Note however that this does not compromise the optimality.

In a first step, we bound the $u_{j}$-decomposition of (3.9) in terms of the $v_{j}$ decomposition of (3.7) with respect to the $\|\cdot\|_{\omega}$ norm in the following lemma. The respective estimate for the $\|\cdot\|_{j, \omega}$ norms then follows with Theorem 2.3.

Lemma 3.4. Let $\omega$ be in the Muckenhoupt class $A_{1}(\Omega)$ and consider a sequence of uniformly refined triangulations $\mathcal{T}_{j}$ and the associated sequence of nested spaces $\mathcal{V}_{j}$ of linear finite elements. The decompositions $v_{j}$ and $u_{j}$ defined in (3.7) and (3.9), respectively, allow for the estimates

$$
\sum_{j=0}^{J} 2^{2 j}\left\|u_{j}\right\|_{\omega}^{2} \leq 4 \sum_{j=0}^{J} 2^{2 j}\left\|v_{j}\right\|_{\omega}^{2}
$$

and

$$
\sum_{j=0}^{J} 2^{2 j}\left\|u_{j}\right\|_{j, \omega}^{2} \leq 4\left(1+C_{B}|\omega|_{A_{1}(\Omega)}^{\frac{1}{2}}\right)^{2}\left(1+6 C_{0}\right)^{2} \sum_{j=0}^{J} 2^{2 j}\left\|v_{j}\right\|_{j, \omega}^{2} .
$$

Proof. Observe that due to (3.9) and (3.2) we have

$$
\begin{aligned}
\left\|u_{j}\right\|_{\omega}^{2} & =\left\|Q_{j}^{\omega} u-Q_{j-1}^{\omega} u\right\|_{\omega}^{2}=\left\langle Q_{j}^{\omega} u-Q_{j-1}^{\omega} u, Q_{j}^{\omega} u\right\rangle_{\omega} \\
& =\left\langle Q_{j}^{\omega} u-Q_{j-1}^{\omega} u, u\right\rangle_{\omega}=\left\langle Q_{j}^{\omega} u-Q_{j-1}^{\omega} u, u-Q_{j-1}^{a} u\right\rangle_{\omega}
\end{aligned}
$$

for all $j$ so that $\left\|u_{j}\right\|_{\omega} \leq\left\|u-Q_{j-1}^{a} u\right\|_{\omega}$ holds for all $j$, and with (3.7) we obtain

$$
\sum_{j=0}^{J} 2^{2 j}\left\|u_{j}\right\|_{\omega}^{2} \leq \sum_{j=0}^{J} 2^{2 j}\left\|u-Q_{j-1}^{a} u\right\|_{\omega}^{2} \leq \sum_{j=0}^{J} 2^{2 j}\left(\sum_{k=j}^{J}\left\|v_{k}\right\|_{\omega}\right)^{2} .
$$

Then using Hardy's inequality (3.13) with $b=2^{2}$ and $a_{k}:=\left\|v_{k}\right\|_{\omega}$, we obtain

$$
\sum_{j=0}^{J} 2^{2 j}\left\|u_{j}\right\|_{\omega}^{2} \leq 4 \sum_{j=0}^{J} 2^{2 j}\left\|v_{j}\right\|_{\omega}^{2}
$$

Passing back from the $\|\cdot\|_{\omega}$ norm to the $\|\cdot\|_{j, \omega}$ norms with the help of Theorem 2.3. we end up with the estimates

$$
\sum_{j=0}^{J} 2^{2 j}\left\|u_{j}\right\|_{\omega}^{2} \leq 4\left(1+6 C_{0}\right)^{2} \sum_{j=0}^{J} 2^{2 j}\left\|v_{j}\right\|_{j, \omega}^{2}
$$


and

$$
\sum_{j=0}^{J} 2^{2 j}\left\|u_{j}\right\|_{j, \omega}^{2} \leq 4\left(1+C_{B}|\omega|_{A_{1}(\Omega)}^{\frac{1}{2}}\right)^{2}\left(1+6 C_{0}\right)^{2} \sum_{j=0}^{J} 2^{2 j}\left\|v_{j}\right\|_{j, \omega}^{2} .
$$

Now, we need to deal with the projection operators $Q_{j}^{a}$ and the associated sequence $v_{j}$ only. Here, we can prove a local estimate for the $\|\cdot\|_{j, \omega}$ norms using a modified version of the duality technique due to Aubin and Nitsche, see also [4, and a certain regularity result for the Neumann problem given in Lemma 3.5 (see the Appendix for a proof). In the following, the space $W_{p}^{k}(\Omega)$ with its associated norm $\|\cdot\|_{k, p, \Omega}$ denotes the classical Sobolev space of $L_{p}(\Omega)$ functions whose first $k$ derivatives are also in $L_{p}(\Omega)$. Similarly, the respective semi-norm is denoted by $|\cdot|_{k, p, \Omega}$.

Lemma 3.5. For any element $T \in \mathcal{T}_{j}$ consider the neighborhood

$$
U:=U_{T}=\bigcup_{\substack{\hat{T} \in \mathcal{T}_{j-1} \\ \hat{T} \subset \sup \left(\psi_{l}^{j-1}\right) \\ \operatorname{supp}\left(\psi_{l}^{j-1}\right) \cap T \neq \emptyset}} \hat{T},
$$

where $\psi_{l}^{j-1}$ denotes the nodal basis function on level $j-1$ centered at node $l$. Let $\tilde{U}$ be such that $\tilde{U} \supset U$ and that the boundary $\partial \tilde{U}$ is smooth. Furthermore, let all vertices $\xi \in \partial U$ also be $\xi \in \partial \tilde{U}$, and $\operatorname{diam}(\tilde{U})<2 \operatorname{diam}(U)$. Let $\tilde{v}_{j}$ denote the continuous and piecewise linear extension of $v_{j}$ from $U$ to $\tilde{U}$ which fulfills

$$
\left\|\tilde{v}_{j}\right\|_{0,2, \tilde{U}} \leq 2\left\|v_{j}\right\|_{0,2, U} .
$$

Then, the solution $\varphi_{U}$ to the inhomogeneous Neumann problem

$$
-\Delta \varphi_{U}=\tilde{v}_{j} \text { in } \tilde{U}, \quad \frac{\partial \varphi_{U}}{\partial \nu}=g \text { on } \partial \tilde{U}
$$

is in $H^{2}(U)$ and allows for the estimate

$$
\left|\varphi_{U}\right|_{2,2, U} \leq \hat{C}\left\|v_{j}\right\|_{0,2, U}
$$

where $\hat{C}$ is essentially the regularity constant $C_{R}$ of (4.1). Here, the boundary data for the Neumann problem are $g:=\alpha h$ with a piecewise linear $h \in L_{2}(\partial \tilde{U})$. Between any two vertices $\xi \in \partial U \cap \partial \tilde{U}$ on the boundary $\partial \tilde{U}$ the function $h \geq 0$ consists of two lines with $h(\xi)=0$ for the vertices $\xi \in \partial U \cap \partial \tilde{U}$. Furthermore, $\alpha \in \mathbb{R}$ is such that the compatibility condition

$$
\int_{\tilde{U}} \tilde{v}_{j}=-\int_{\partial \tilde{U}} g
$$

is fulfilled.

With this lemma, we are in a position to prove the following local inequality for the level-dependent norms $\|\cdot\|_{j, \omega}$ in Theorem 3.6. However, we can obtain this estimate on levels $j \geq j_{0}$ with $j_{0}=j_{0}(\omega)$ independent of $J$ only. Note that the introduction of the additional parameter $j_{0}$ does not compromise the optimality. For weights $\omega \in A_{1}(\Omega)$ we can determine $j_{0}$ easily from the limited growth condition (3.19) given below. For many practically relevant diffusion coefficients we find $j_{0}$ to be rather small; see section 3.3 . 
Theorem 3.6. Let $\omega$ be in the Muckenhoupt class $A_{1}(\Omega)$ and consider a sequence of uniformly refined triangulations $\mathcal{T}_{j}$ and the associated sequence of nested spaces $\mathcal{V}_{j}$ of linear finite elements. Let $j$ be large enough, i.e., $j \geq j_{0}$ where $j_{0}$ is given by the smallest value such that

$$
|\omega|_{A_{1}(\Omega)}^{\frac{1}{2}} \leq \frac{1}{2} \frac{2^{j_{0}-2}}{4 C^{*} \sqrt{C_{3}}}
$$

holds with

$$
C_{3}:=\max _{S, S^{\prime} \in \mathcal{T}_{0}} \frac{\mu(S)}{\mu\left(S^{\prime}\right)} \quad \text { and } \quad C^{*}=\max \left\{C_{H}, 8 \sqrt{C_{3}} \hat{C}\right\}
$$

Then the estimate

$$
\left\|v_{j}\right\|_{j, \omega, U} \leq C_{L}|\omega|_{A_{1}(\Omega)}^{\frac{1}{2}} \operatorname{diam}(U)\left\|\nabla v_{j}\right\|_{j, \omega, U} \text { with } C_{L}:=8 C^{*} \sqrt{C_{3}} C_{1}
$$

holds with respect to the neighborhood $U=U_{T}$ given in (3.15) for $v_{j}$ defined in (3.7).

Proof. we note that we can assume $v_{j}(x) \geq 0$ in $U$ since otherwise $v_{j}$ changes sign in $U$ and we can obtain the desired inequality (3.20) directly. Therefore in the following we need to consider only those $U$ which do not intersect boundary elements $T \in \mathcal{T}_{j-1}$.

With the help of (2.6) and Lemma 3.5 we obtain

$$
\begin{aligned}
\left\|v_{j}\right\|_{j, \omega, U}^{2} & =-\int_{U} \omega v_{j} \Delta \varphi_{U} \\
& =\underbrace{\sum_{S \subset U} \omega_{S} \int_{S} \nabla v_{j} \nabla \varphi_{U}}_{:=I_{1}(U)}-\underbrace{\sum_{S \subset U} \omega_{S} \int_{\partial S} v_{j} \nabla \varphi_{U} \cdot n_{\partial S}}_{:=I_{2}(U)}
\end{aligned}
$$

after integration by parts on each $S \subset U$. Concerning the first sum $I_{1}(U)$, observe that by definition (3.7) of $v_{j}$

$$
0=a_{j}\left(v_{j}, w\right)=a\left(v_{j}, w\right)=\sum_{S \in \mathcal{T}_{j}} \omega_{S} \int_{S} \nabla v_{j} \nabla w
$$

holds for all $w \in \mathcal{V}_{j-1}$. Now, we choose $w$ to be the function in $\mathcal{V}_{j-1}$ which interpolates $\varphi_{U}$ at the nodes in $T$ and has support in $U=U_{T}$. Then, we can estimate $I_{1}(U)$ via the Bramble-Hilbert Lemma on $U$

$$
\begin{aligned}
\left|I_{1}(U)\right| & =\left|\sum_{S \subset U} \omega_{S} \int_{S} \nabla \varphi_{U} \nabla v_{j}\right|=\left|\sum_{S \subset U} \omega_{S} \int_{S} \nabla\left(\varphi_{U}-w\right) \nabla v_{j}\right| \\
& \leq\left(\sum_{S \subset U} \omega_{S} \int_{S}\left|\nabla\left(\varphi_{U}-w\right)\right|^{2}\right)^{\frac{1}{2}}\left\|\nabla v_{j}\right\|_{j, \omega, U} \\
& \leq C_{H} \operatorname{diam}(U)\left|\varphi_{U}\right|_{2,2, U}\left(\max _{S \subset U} \sqrt{\omega_{S}}\right)\left\|\nabla v_{j}\right\|_{j, \omega, U},
\end{aligned}
$$

where the constant $C_{H}$ depends only on the shape of $U$, i.e., by assumption only on the initial triangulation $\mathcal{T}_{0}$.

The second sum $I_{2}(U)$ in (3.21) can be estimated by

$$
\left|I_{2}(U)\right|=\left|\sum_{S \subset U} \omega_{S} \int_{\partial S} v_{j}\left(\nabla \varphi_{U}, n_{\partial S}\right)\right| \leq \sum_{S \subset U} \omega_{S}\left\|v_{j}\right\|_{\infty, \partial S} \int_{\partial S}\left|\nabla \varphi_{U}\right|
$$


where $\|\cdot\|_{\infty, \partial S}$ denotes the $L_{\infty}(\partial S)$ norm. Note that due to the choice of $g$ in Lemma 3.5 the normal derivative $\partial \varphi_{U} / \partial \nu_{\xi}=g$ vanishes at each vertex $\xi \in \partial U$. Also, since any normal vector $n_{K}$ of an arbitrary edge $K \subset U$ can be represented as a linear combination of three such normal vectors at $\xi_{i}, i=1,2,3$, we can bound the normal derivative at $x \in K$ by

$$
\begin{aligned}
\left|\left(\nabla \varphi_{U}, n_{K}\right)(x)\right| & =\left|\sum_{i=1}^{3} \beta_{i} \frac{\partial \varphi_{U}}{\partial \nu_{\xi_{i}}}(x)\right| \\
& =\left|\sum_{i=1}^{3} \beta_{i}\left(\frac{\partial \varphi_{U}}{\partial \nu_{\xi_{i}}}(x)-\frac{\partial \varphi_{U}}{\partial \nu_{\xi_{i}}}\left(\xi_{i}\right)\right)\right| \\
& \leq \sum_{i=1}^{3}\left|\beta_{i}\right| \int_{0}^{1}\left|\left(\nabla \frac{\partial \varphi_{U}}{\partial \nu_{\xi_{i}}}\right)\left(\xi_{i}+t\left(x-\xi_{i}\right), x-\xi_{i}\right) d t\right|
\end{aligned}
$$

with $\sum_{i=1}^{3}\left|\beta_{i}\right|^{2}=1$. Hence after integration over the edge $K$ we obtain

$$
\int_{K}\left|\left(\nabla \varphi_{U}, n_{K}\right)(x)\right| d x \leq \operatorname{diam}(U) \sum_{|\alpha|=2} \int_{U}\left|D^{\alpha} \varphi_{U}\right| d x \leq \operatorname{diam}(U)(\mu(U))^{\frac{1}{2}}\left|\varphi_{U}\right|_{2,2, U} .
$$

Altogether, we can now establish the estimate

$$
\begin{aligned}
\left|I_{2}(U)\right| & \leq 2 \sum_{S \subset U} \omega_{S}\left\|v_{j}\right\|_{\infty, \partial S} \operatorname{diam}(U)(\mu(U))^{\frac{1}{2}}\left|\varphi_{U}\right|_{2,2, U} \\
& \leq 8 \sqrt{C_{3}}\left\|v_{j}\right\|_{j, \omega, U}\left(\max _{S \subset U} \sqrt{\omega_{S}}\right) \operatorname{diam}(U)\left|\varphi_{U}\right|_{2,2, U},
\end{aligned}
$$

due to $\sum_{S \subset U} \sqrt{\omega_{S}}(\mu(U))^{\frac{1}{2}}\left\|v_{j}\right\|_{\infty, \partial S} \leq 4 \sqrt{C_{3}}\left\|v_{j}\right\|_{j, \omega, U}$ since $\mu(\mathrm{U}) \leq 16 C_{3} \mu(S)$. The assertion (3.20) then follows easily with the aid of (3.17): Insert (3.17) into (3.22) and (3.23) and obtain

$$
\begin{aligned}
\left\|v_{j}\right\|_{j, \omega, U}^{2} & \leq\left|I_{1}(U)\right|+\left|I_{2}(U)\right| \\
& \leq C^{*} \operatorname{diam}(U)\left(\left\|v_{j}\right\|_{j, \omega, U}+\left\|\nabla v_{j}\right\|_{j, \omega, U}\right)\left(\max _{S \subset U} \sqrt{\omega_{S}}\right)\left\|v_{j}\right\|_{0,2, U}
\end{aligned}
$$

with $C^{*}:=\max \left\{C_{H}, 8 \sqrt{C_{3}} \hat{C}\right\}$. With the estimate $\left(\max _{S \subset U} \sqrt{\omega_{S}}\right)\left\|v_{j}\right\|_{0,2, U} \leq$ $\left(\max _{S^{\prime}, S \subset U} \sqrt{\omega_{S} / \omega_{S^{\prime}}}\right)\left\|v_{j}\right\|_{j, \omega, U}$, this yields the inequality

$$
\left\|v_{j}\right\|_{j, \omega, U} \leq C^{*} \operatorname{diam}(U)\left(\max _{S^{\prime}, S \subset U} \sqrt{\frac{\omega_{S}}{\omega_{S^{\prime}}}}\right)\left(\left\|v_{j}\right\|_{j, \omega, U}+\left\|\nabla v_{j}\right\|_{j, \omega, U}\right)
$$

after division by $\left\|v_{j}\right\|_{j, \omega, U}$. Due to the use of uniformly refined triangulations we have

$$
\frac{\omega_{S}}{\omega_{S^{\prime}}}=\frac{\mu\left(S^{\prime}\right)}{\mu(S)} \frac{\int_{S} \omega}{\int_{S^{\prime}} \omega} \leq C_{3} \frac{\int_{S} \omega}{\int_{S^{\prime}} \omega} \leq \frac{\mu(U)}{\mu(S)} \frac{\omega_{U}}{\omega_{S^{\prime}}} \leq 16 C_{3}|\omega|_{A_{1}(\Omega)}
$$

since $\omega \in A_{1}(\Omega)$. Hence, we obtain

$$
\left(1-4 C^{*} \sqrt{C_{3}|\omega|_{A_{1}(\Omega)}} \operatorname{diam}(U)\right)\left\|v_{j}\right\|_{j, \omega, U} \leq 4 C^{*} \sqrt{C_{3}|\omega|_{A_{1}(\Omega)}} \operatorname{diam}(U)\left\|\nabla v_{j}\right\|_{j, \omega, U}
$$


With the assumption (3.19) and $\operatorname{diam}(U) \leq C_{1} 2^{-j+2}$ this yields the asserted inequality

$$
\begin{aligned}
\left\|v_{j}\right\|_{j, \omega, U} & \leq \frac{4 C^{*} \sqrt{C_{3}|\omega|_{A_{1}(\Omega)}}}{\left(1-4 C^{*} \sqrt{C_{3}|\omega|_{A_{1}(\Omega)}} \operatorname{diam}(U)\right)} \operatorname{diam}(U)\left\|\nabla v_{j}\right\|_{j, \omega, U} \\
& \leq 8 C^{*} \sqrt{C_{3}|\omega|_{A_{1}(\Omega)}} \operatorname{diam}(U)\left\|\nabla v_{j}\right\|_{j, \omega, U} .
\end{aligned}
$$

A direct consequence of Theorem 3.6 are the following lower bounds.

Theorem 3.7. Let $\omega$ be in the Muckenhoupt class $A_{1}(\Omega)$ and consider a sequence of uniformly refined triangulations $\mathcal{T}_{j}$ and the associated sequence of nested spaces $\mathcal{V}_{j}$ of linear finite elements. Consider the set $U=U_{T}$ defined in (3.15) for every $T \in \mathcal{T}_{j-1}$. Let $j \geq j_{0}$ so that (3.19) is satisfied and Theorem 3.6 is applicable. Consider the decomposition $u_{j}$ defined in (3.9) for $u \in \mathcal{V}_{J}$. Then there hold the estimates

$$
\sum_{j=j_{0}}^{J} 2^{2 j}\left\|u_{j}\right\|_{\omega}^{2} \leq 4\left(1+6 C_{0}\right)^{2} C_{L}^{2}|\omega|_{A_{1}(\Omega)} a(u, u)
$$

and

$$
\sum_{j=j_{0}}^{J} 2^{2 j}\left\|u_{j}\right\|_{j, \omega}^{2} \leq 4\left(1+C_{B}|\omega|_{A_{1}(\Omega)}^{\frac{1}{2}}\right)^{2}\left(1+6 C_{0}\right)^{2} C_{L}^{2}|\omega|_{A_{1}(\Omega)} a(u, u) .
$$

Proof. Using Lemma 3.4, we establish the estimate (3.14),

$$
\begin{aligned}
\sum_{j=j_{0}}^{J} 2^{2 j}\left\|u_{j}\right\|_{\omega}^{2} & \leq 4\left(1+6 C_{0}\right)^{2} \sum_{j=j_{0}}^{J} 2^{2 j}\left\|v_{j}\right\|_{j, \omega}^{2} \\
& \leq 4\left(1+6 C_{0}\right)^{2} C_{L}^{2}|\omega|_{A_{1}(\Omega)} \sum_{j=j_{0}}^{J} a\left(v_{j}, v_{j}\right)
\end{aligned}
$$

after squaring (3.20) and the summation with respect to $U_{T}$, i.e., over all $T \in \mathcal{T}_{j-1}$. Then, with (3.8) and $a\left(v_{j}, v_{j}\right)=\left\|\nabla v_{j}\right\|_{j, \omega}^{2}$ the assertion (3.24) follows. We obtain (3.25) with the help of the left-hand side inequality of Theorem 2.3.

Finally, let us consider the remainder terms for the coarser levels $0 \leq j<j_{0}$. Since $j_{0}$ is independent of $J$ it is sufficient to establish the following equivalence which explicitly involves $j_{0}$ in the following lemma.

Lemma 3.8. Let $\omega$ be in the Muckenhoupt class $A_{1}(\Omega)$ and consider a sequence of uniformly refined triangulations $\mathcal{T}_{j}$ and the associated sequence of nested spaces $\mathcal{V}_{j}$ of linear finite elements. Then the estimates

$$
\sum_{j=0}^{j_{0}-1} 2^{2 j}\left\|v_{j}\right\|_{j, \omega}^{2} \leq 4^{j_{0}}|\omega|_{A_{1}(\Omega)} C_{4} C_{5} \sum_{j=0}^{j_{0}-1} a\left(v_{j}, v_{j}\right)
$$

and

$$
\sum_{j=0}^{j_{0}-1} 2^{2 j}\left\|v_{j}\right\|_{\omega}^{2} \leq 4^{j_{0}}|\omega|_{A_{1}(\Omega)} C_{4} C_{5}\left(1+6 C_{0}\right)^{2} \sum_{j=0}^{j_{0}-1} a\left(v_{j}, v_{j}\right)
$$


hold for the decomposition $v_{j}$ defined in (3.7) for $u \in \mathcal{V}_{J}$ with constants $C_{4}$ and $C_{5}$ depending on the initial triangulation only.

Proof. Let $\mathcal{S}_{T}=\left\{S_{i} \in \mathcal{T}_{j}, i=1, \ldots, m\right\}$ denote the shortest chain of triangles $S \in \mathcal{T}_{j}$ which connect a triangle $T \in \mathcal{T}_{j}$ to the boundary $\partial \Omega$. For any $x \in T$ consider the sequence of points $\left\{x_{i}\right\}_{i=0}^{m+1}$ with $x_{0}=x, x_{m+1} \in \partial \Omega$, and $x_{i}, x_{i+1} \in \partial S_{i}$ for $i=1, \ldots, m$ which connect $x$ to the boundary $\partial \Omega$. Recall that $v_{j}$ vanishes on the boundary $\partial \Omega$, hence we have the point-wise estimate

$$
\left|v_{j}(x)\right|=\left|\sum_{i=0}^{m} x_{i+1}-x_{i}\right| \leq \sum_{i=0}^{m}\left|x_{i+1}-x_{i}\right| \leq\left\|\left.\nabla v\right|_{S_{i}}\right\| \operatorname{diam}\left(S_{i}\right) .
$$

This yields

$$
\begin{aligned}
\left\|v_{j}\right\|_{j, \omega}^{2} & =\sum_{T \in \mathcal{T}_{j}} \int_{T} \omega(x)\left|v_{j}(x)\right|^{2} d x \leq \sum_{T \in \mathcal{T}_{j}} \omega_{T} \mu(T)\left(\sum_{S \in \mathcal{S}_{T}} \operatorname{diam}(S)\left\|\left.\nabla v_{j}\right|_{S}\right\|\right)^{2} \\
& \leq \sum_{T \in \mathcal{T}_{j}} \omega_{T} \mu(T) \sum_{S^{\prime} \in \mathcal{S}_{T}} \operatorname{diam}\left(S^{\prime}\right) \sum_{S \in \mathcal{S}_{T}} \operatorname{diam}(S)\left\|\left.\nabla v_{j}\right|_{S}\right\|^{2} \\
& \leq|\omega|_{A_{1}(\Omega)} C_{4} \sum_{T \in \mathcal{T}_{j}} \sum_{S \in \mathcal{S}_{T}} \operatorname{diam}(S) \omega_{S} \mu(S)\left\|\left.\nabla v_{j}\right|_{S}\right\|^{2}
\end{aligned}
$$

due to the fact that $\sum_{S \in \mathcal{S}_{T}} \operatorname{diam}(S) \leq C \operatorname{diam}(\Omega)=: C_{4}$. Interchanging the summation and counting multiples of a triangle $S$ by $M_{S}$, we obtain

$$
\left\|v_{j}\right\|_{j, \omega}^{2} \leq|\omega|_{A_{1}(\Omega)} C_{4} \sum_{S \in \mathcal{T}_{j}} M_{S} \operatorname{diam}(S) \int_{S} \omega\left\|\nabla v_{j}\right\|^{2} .
$$

The number $M_{S}$ gives the number of chains $\mathcal{S}_{T}$ for any $T \in \mathcal{T}_{j}$ that contain the triangle $S$. It is obvious that $M_{S}$ is larger for triangles $S$ closer to the boundary. However, it is clear that $M_{S}$ is bounded by the number of triangles intersected by the diameter. Hence, we have $M_{S} \operatorname{diam}(S) \leq C \operatorname{diam}(\Omega)=: C_{5}$ due to the uniformity of the triangles. This leads to the estimate

$$
\left\|v_{j}\right\|_{j, \omega}^{2} \leq|\omega|_{A_{1}(\Omega)} C_{4} C_{5} \sum_{S \in \mathcal{T}_{j}} \int_{S} \omega\left\|\nabla v_{j}\right\|^{2}=|\omega|_{A_{1}(\Omega)} C_{4} C_{5} a\left(v_{j}, v_{j}\right)
$$

and we finally obtain the assertion

$$
\sum_{j=0}^{j_{0}-1} 2^{2 j}\left\|v_{j}\right\|_{j, \omega}^{2} \leq 4^{j_{0}}|\omega|_{A_{1}(\Omega)} C_{4} C_{5} \sum_{j=0}^{j_{0}-1} a\left(v_{j}, v_{j}\right) .
$$

The corresponding estimate for the $\|\cdot\|_{\omega}$ norm is obtained by the right-hand inequality of Theorem 2.3 .

Altogether, we can now establish our robust and optimal norm equivalencies in the following theorem which summarizes the results of Theorems 3.3 and 3.7 as well as of Lemma 3.8. Note that we make use of the fact that $4^{j_{0}}=2 C_{L}|\omega|_{A_{1}(\Omega)}^{\frac{1}{2}}$ which stems from the limited growth condition (3.19), i.e.,

$$
j_{0}=\ln \left(C_{L}|\omega|_{A_{1}(\Omega)}^{\frac{1}{2}}\right)
$$

to eliminate $j_{0}$ from the norm equivalencies. However, (3.19) must be satisfied for some $j_{0}<J$. 
Theorem 3.9. Let $\omega$ be in the Muckenhoupt class $A_{1}(\Omega)$ such that (3.19) holds for a $j_{0}<J$. Consider a sequence of uniformly refined triangulations $\mathcal{T}_{j}$ and the associated sequence of nested spaces $\mathcal{V}_{j}$ of linear finite elements. Then the equivalence

$$
K_{U} a(u, u) \leq \sum_{j=0}^{J} 2^{2 j}\left\|u_{j}\right\|_{j, \omega}^{2} \leq K_{L} a(u, u)
$$

and the equivalence

$$
K_{\tilde{U}} a(u, u) \leq \sum_{j=0}^{J} 2^{2 j}\left\|u_{j}\right\|_{\omega}^{2} \leq K_{\tilde{L}} a(u, u)
$$

hold for $u \in \mathcal{V}_{J}$ and its associated decomposition $u_{j}$ defined in (3.9), where the constants are given by

$$
\begin{aligned}
K_{U} & :=\left(C_{U}^{2}|\omega|_{A_{1}(\Omega)}\right)^{-1} \\
K_{L} & :=\left(4\left(1+6 C_{0}\right)^{2} C_{L}^{2}|\omega|_{A_{1}(\Omega)}\left(1+C_{B}|\omega|_{A_{1}(\Omega)}^{\frac{1}{2}}\right)^{2}+2 C_{L}|\omega|_{A_{1}(\Omega)}^{\frac{3}{2}} C_{4} C_{5}\right), \\
K_{\tilde{U}} & :=\left(C_{U}^{2}|\omega|_{A_{1}(\Omega)}\left(1+C_{B}|\omega|_{A_{1}(\Omega)}^{\frac{1}{2}}\right)^{2}\right)^{-1} \\
K_{\tilde{L}} & :=\left(4\left(1+6 C_{0}\right)^{2} C_{L}^{2}|\omega|_{A_{1}(\Omega)}+\left(1+6 C_{0}\right)^{2} 2 C_{L}|\omega|_{A_{1}(\Omega)}^{\frac{3}{2}} C_{4} C_{5}\right) .
\end{aligned}
$$

3.3. Examples. Finally, we consider a few examples of weight functions $\omega$ for which our theory holds. First of all, there is a close connection between the Muckenhoupt class $A_{1}(\Omega)$ with the space $\operatorname{BMO}(\Omega)$ via the implication

$$
\omega \in A_{1}(\Omega) \quad \Rightarrow \quad \ln (\omega) \in \operatorname{BMO}(\Omega) .
$$

Let us consider a weight function $\omega$ with $\inf _{x \in \Omega} \omega(x)=m_{\omega}>0$ and $\sup _{x \in \Omega} \omega(x)$ $=M_{\omega}$. Then there holds for all balls $B \subset \Omega$ the inequality

$$
\frac{\left\|\omega^{-1}\right\|_{L_{\infty}(\Omega)}}{\mu(B)} \int_{B} \omega=\frac{m_{\omega}^{-1}}{\mu(B)} \int_{B} \omega \leq \frac{M_{\omega}}{m_{\omega}} .
$$

Hence, any positive piecewise constant function $\omega$ is in $A_{1}(\Omega)$. Let us now assume that $m_{\omega}=1$ and $M_{\omega}=\epsilon^{-1}$, i.e., it is suffcient to assume a maximal jump of height $\epsilon^{-1}$. Then we obtain a minimal refinement level

$$
j_{0} \approx \ln \left(\epsilon^{-1}\right) \text {. }
$$

Thus $j_{0}$ is a rather small number even for very large jumps. Note that we do not require the jumps to be aligned with the mesh on any level, i.e., no mesh must resolve the jumps. There is no restriction on the frequency or the location of the jumps.

\section{Concluding Remarks}

We presented two optimal and robust norm equivalencies based on certain weighted norms for diffusion problems $-\nabla \omega \nabla u=f$ in two space dimensions with a scalar diffusion coefficient $\omega$. We only require $\omega$ to be in the Muckenhoupt class $A_{1}(\Omega)$ 
to obtain our optimal bounds. This covers all piecewise constant functions independent of the location of jumps, their number or their frequency. In contrast to previous results, we do not require the resolution of the jumps on a particular level, i.e., the coarsest level. However, the constants of our norm equivalence involve the height of the maximal jump and thus for all practical purposes it is necessary to assume $|\omega|_{A_{1}(\Omega)}$ to be small.

\section{APPENDIX}

Proof of Lemma 3.5. Consider the scaled domain $\hat{\Omega}:=R \tilde{U}$ and the spaces

$$
\begin{aligned}
V(\hat{\Omega}) & :=\left\{\varphi \in W_{2}^{2}(\hat{\Omega}): \int_{\hat{\Omega}} \varphi d x=0\right\} \quad \text { and } \\
W(\hat{\Omega}) & :=\left\{\langle f, g\rangle \in L_{2}(\hat{\Omega}) \times W_{2}^{\frac{1}{2}}(\partial \hat{\Omega}): \int_{\hat{\Omega}} f d x+\int_{\partial \hat{\Omega}} g d s=0\right\}
\end{aligned}
$$

and the mapping $T: \varphi \in V \mapsto T \varphi \in W$ defined by

$$
T \varphi:= \begin{cases}f=\Delta \varphi & \in L_{2}(\hat{\Omega}), \\ g=\partial \varphi / \partial \nu & \in W_{2}^{\frac{1}{2}}(\partial \hat{\Omega}) .\end{cases}
$$

This mapping is linear and continuous; i.e., there exists a constant $M_{\hat{\Omega}}$ such that

$$
\|T \varphi\|_{W} \equiv\|f\|_{0,2, \hat{\Omega}}+\|g\|_{1 / 2,2, \partial \hat{\Omega}} \leq M_{\hat{\Omega}}\|\varphi\|_{2,2, \hat{\Omega}}
$$

holds. Furthermore, $T$ is also bijective from $V$ onto $W$; see [23], pp. 336-339. Hence by the open mapping theorem its inverse $T^{-1}:\langle f, g\rangle \mapsto \varphi$ is also continuous and satisfies

$$
\|\varphi\|_{V} \equiv\|\varphi\|_{2,2, \hat{\Omega}} \leq L_{\hat{\Omega}}\left(\|\Delta \varphi\|_{0,2, \hat{\Omega}}+\|\partial \varphi / \partial \nu\|_{1 / 2,2, \partial \hat{\Omega}}\right), \quad \varphi \in W(\hat{\Omega}),
$$

with a constant $L_{\hat{\Omega}}$. However, it is still necessary to determine the dependence of $L_{\hat{\Omega}}$ on the size of $\hat{\Omega}=R \tilde{U}$, i.e., on the scaling $R$, since by definition (3.15) $U=U_{T}$ depends on the level $j$. To this end, let us consider the scaling $R$ such that $R^{-1}:=\operatorname{diam}(\tilde{U}) \leq 2 \operatorname{diam}(U)$ so that

$$
\|\psi\|_{2,2, \hat{\Omega}} \leq C_{R}\left(\|\Delta \psi\|_{0,2, \hat{\Omega}}+\|\partial \psi / \partial \nu\|_{1 / 2,2, \partial \hat{\Omega}}\right), \quad \psi \in W(\hat{\Omega}),
$$

with $C_{R}$ depending only on the shape of $U$ but not on the size. Hence, $C_{R}$ depends only on the initial triangulation $\mathcal{T}_{0}$. The connection between $\varphi$ and $\psi$ is given by $\psi(t)=\varphi(t / R):=\varphi(x)$. Therefore, there hold the equivalencies

$$
\|\psi\|_{0,2, \hat{\Omega}}=R\|\varphi\|_{0,2, \tilde{U}}, \quad|\psi|_{1,2, \hat{\Omega}}=|\varphi|_{1,2, \tilde{U}}, \quad \text { and } \quad|\psi|_{2,2, \hat{\Omega}}=R^{-1}|\varphi|_{2,2, \tilde{U}} .
$$

Using the explicit form of the trace norm $\|\cdot\|_{1 / 2,2, \partial \hat{\Omega}}$ (see e.g. [23], p. 94) given by

$$
\begin{aligned}
\|\psi\|_{1 / 2,2, \partial \hat{\Omega}}^{2} & :=\sum_{i}\left\|\psi_{i}\right\|_{0,2, \partial \hat{\Omega}}^{2}+\sum_{i}\left|\psi_{i}\right|_{1 / 2,2, \partial \hat{\Omega}}^{2} \\
\left|\psi_{i}\right|_{1 / 2,2, \partial \hat{\Omega}}^{2} & :=\int_{\partial \hat{\Omega}} \int_{\partial \hat{\Omega}} \frac{\left|\psi_{i}(t)-\psi_{i}(s)\right|^{2}}{|t-s|^{2}} d \sigma d \sigma,
\end{aligned}
$$

where $\psi=\sum_{i} \psi_{i}$ is a partition of $\psi$ with respect to the representation by charts of the curve $\partial \hat{\Omega}$ with curve element $d \sigma$, we conclude that

$$
\|\partial \psi / \partial \nu\|_{1 / 2,2, \partial \hat{\Omega}}=R^{-1 / 2}\|\partial \varphi / \partial \nu\|_{0,2, \partial \tilde{U}}+R^{-1}|\partial \varphi / \partial \nu|_{1 / 2,2, \partial \tilde{U}}
$$


Consequently, by (4.1) we obtain the estimate

$$
\begin{aligned}
\left|\varphi_{U}\right|_{1,2, \tilde{U}}+\operatorname{diam}(U)\left|\varphi_{U}\right|_{2,2, \tilde{U}} \leq 2 C_{R} \operatorname{diam}(U) & \left(\left\|\tilde{v}_{j}\right\|_{0,2, \tilde{U}}\right. \\
& \left.+|g|_{1 / 2,2, \partial \tilde{U}}+\frac{\|g\|_{0,2, \partial \tilde{U}}}{\sqrt{\operatorname{diam}(U)}}\right)
\end{aligned}
$$

for the data of the Neumann problem above, i.e., $\varphi=\varphi_{U} \in W_{2}^{2}(\tilde{U}),-\Delta \varphi_{U}=\tilde{v}_{j}$ in $\tilde{U}$ and $\partial \varphi / \partial \nu=g$ on $\partial \tilde{U}$. Now we use the fact that $g$ is piecewise linear on $\partial \tilde{U}$ by construction. Therefore,

$$
\begin{aligned}
|g|_{1 / 2,2, \partial \tilde{U}} & \leq C(\operatorname{diam}(U))^{-1}\|g\|_{0,1, \partial \tilde{U}} \\
\|g\|_{0,2, \partial \tilde{U}} & \leq C(\operatorname{diam}(U))^{-1 / 2}\|g\|_{0,1, \partial \tilde{U}}
\end{aligned}
$$

holds with a constant $C$ independent of $\tilde{U}$ and $g$. For completeness, we give the proof of the inequalities (4.4) and (4.5) here. To this end, let $\partial \tilde{U}_{i}$ be one of the two pieces of the segment of $\partial \tilde{U}$ between two vertices of $\mathcal{T}_{j-1}$. Here $g$ is a linear function. Then by (4.2) with $\psi_{i}=g$

$$
|g|_{1 / 2,2, \partial \tilde{U}_{i}}=\left(\int_{\partial \tilde{U}_{i}} \int_{\partial \tilde{U}_{i}} \frac{|g(t)-g(s)|^{2}}{|t-s|^{2}} d \sigma d \sigma\right)^{\frac{1}{2}} \leq \operatorname{diam}\left(\partial \tilde{U}_{i}\right)|\nabla g|_{\infty, \partial \tilde{U}_{i}}
$$

follows. Furthermore, we can replace $g$ by $\tilde{g}:=g-\bar{g}$ where $\bar{g}:=\min _{\partial \tilde{U}_{i}}|g|$. Then $\tilde{g}$ has a zero on $\partial \tilde{U}_{i}$ and we have the equivalence

$$
\int_{\partial \tilde{U}_{i}}|\tilde{g}| d \sigma \asymp\left(\operatorname{diam}\left(\partial \tilde{U}_{i}\right)\right)^{2}|\nabla g|_{\infty, \partial \tilde{U}_{i}},
$$

with constants independent of $\tilde{U}_{i}$ and $g$. Since

$$
\int_{\partial \tilde{U}_{i}}|\tilde{g}| d \sigma \leq \int_{\partial \tilde{U}_{i}}|g| d \sigma+\operatorname{diam}\left(\partial \tilde{U}_{i}\right) \bar{g} \leq 2 \int_{\partial U_{i}}|g| d \sigma=2\|g\|_{0,1, \partial \tilde{U}_{i}},
$$

the comparison with the previous inequality yields (4.4). The second inequality (4.5) follows from

$$
\begin{aligned}
\int_{\partial \tilde{U}_{i}}|g|^{2} d \sigma & \leq 2\left(\int_{\partial \tilde{U}_{i}}|\tilde{g}|^{2} d \sigma+\int_{\partial \tilde{U}_{i}}|\bar{g}|^{2} d \sigma\right) \\
& \left.\leq 2\left(\operatorname{diam}\left(\partial \tilde{U}_{i}\right)\right)^{3}|\nabla g|_{\infty, \partial \tilde{U}_{i}}^{2}+\left(\operatorname{diam}\left(\partial \tilde{U}_{i}\right)\right)^{-1}\left(\int_{\partial \tilde{U}_{i}}|g| d \sigma\right)^{2}\right) \\
& \leq C\left(\operatorname{diam}\left(\partial \tilde{U}_{i}\right)\right)^{-1}\left(\int_{\partial \tilde{U}_{i}}|g| d \sigma\right)^{2}
\end{aligned}
$$

where we have used (4.6) in the last step. This completes the proof of (4.4) and (4.5), since we can choose $\tilde{U}$ such that $\operatorname{diam}(\tilde{U}) \asymp \operatorname{diam}(\partial \tilde{U})$.

Inserting (4.4) and (4.5) into inequality (4.3) and taking (3.16) and (3.18) into account, the desired inequality (3.17) directly follows as

$$
\begin{aligned}
\left|\varphi_{U}\right|_{2,2, U} & \leq \hat{C}\left(\left\|\tilde{v}_{j}\right\|_{0,2, \tilde{U}}+(\operatorname{diam}(U))^{-1}\|g\|_{0,1, \partial \tilde{U}}\right) \\
& =\hat{C}\left(\left\|\tilde{v}_{j}\right\|_{0,2, \tilde{U}}+(\operatorname{diam}(U))^{-1}\left\|\tilde{v}_{j}\right\|_{0,1, \tilde{U}}\right) \leq \hat{C}\left\|v_{j}\right\|_{0,2, U}
\end{aligned}
$$




\section{REFERENCES}

1. R. E. Alcouffe, A. Brandt, J. E. Dendy and J. W. Painter, The Multi-Grid Method for the Diffusion Equation with Strongly Discontinuous Coefficients, SIAM J. Sci. Comput. 2 (1981), 430-454. MR639011 (82k:65072)

2. S. Beuchler, R. Schneider, and C. Schwab, Multiresolution Weighted Norm Equivalences and Applications, Tech. Report 02-09, Prepreint-Reihe Sonderforschungsbereich 393 TU Chemnitz, 2002.

3. F. Bornemann and H. Yserentant, A Basic Norm Equivalence for the Theory of Multilevel Methods, Numer. Math. 64 (1993), 455-476. MR.1213412 (94b:65155)

4. D. Braess, Finite Elements: Theory, Fast Solvers, and Applications in Solid Mechanics, Cambridge University Press, 2001. MR.1827293 (2001k:65002)

5. J. H. Bramble and Jinchao Xu, Some estimates for a weighted $L^{2}$ projection, Mathematics of Computation 56 (1991), 463-476. MR1066830(91k:65140)

6. A. Brandt, Multi-Level Adaptive Technique (MLAT) for Fast Numerical Solution to Boundary Value Problems, Proc. of the Third Int. Conf. on Numerical Methods in Fluid Mechanics, Univ. Paris 1972 (New York, Berlin, Heidelberg) (H. Cabannes and R. Teman, eds.), Springer, 1973.

7. _ Multi-Level Adaptive Solutions to Boundary-Value Problems, Math. Comp. 31 (1977), 333-390. MR0431719 (55:4714)

8. _ Algebraic Multigrid Theory: The Symmetric Case, Preliminary Proceedings for the International Multigrid Conference (Copper Mountain, Colorado), April 1983.

9. _ Algebraic Multigrid Theory: The Symmetric Case, Appl. Math. Comput. 19 (1986), 23-56. MR849831 (87j:65042)

10. A. Brandt, S. F. McCormick, and J. W. Ruge, Algebraic Multigrid for Automatic Multigrid Solutions with Application to Geodetic Computations, Technical Report, Institute for Computational Studies, Fort Collins, Colorado, October 1982.

11. _ Algebraic Multigrid for Sparse Matrix Equations, Sparsity and Its Applications (D. J. Evans, ed.), Cambridge University Press, 1984.

12. J. E. Dendy, Black Box Multigrid, J. Comput. Phys. 48 (1982), 366-386. MR684260 (84b:65097)

13. M. Dryja, M. V. Sarkis, and O. B. Widlund, Multilevel Schwarz Methods for Elliptic Problems with Discontinuous Coefficients in Three Dimensions, Numer. Math. 72 (1996), 313-348. MR 1367653 (96h:65134)

14. T. Grauschopf, M. Griebel, and H. Regler, Additive Multilevel-Preconditioners based on Bilinear Interpolation, Matrix Dependent Geometric Coarsening and Algebraic Multigrid Coarsening for Second Order Elliptic PDEs, Applied Numerical Mathematics 23(1) (1997), 63-96. MR 1438081 (97j:65193)

15. W. Hackbusch, Ein iteratives Verfahren zur schnellen Auflösung elliptischer Randwertprobleme, Tech. Report 76-12, Mathematisches Institut, Universität zu Köln, 1976.

16. _ـ A Fast Numerical Method for Elliptic Boundary Value Problems with Variable Coefficients, 2nd GAMM-Conf. Numer. Meth. Fl. Mech. (Köln) (E. H. Hirschel and W. Geller, eds.), Deutsche Forschungs- und Versuchsanstalt für Luft- und Raumfahrt, 1977, pp. 50-57.

17. Multi-grid methods and applications, Springer, 1985.

18. P. Oswald, On the Robustness of the BPX-Preconditioner with Respect to Jumps in the Coefficients, Math. Comp. 68 (1999), 633-650. MR1620239 (99i:65143)

19. J. W. Ruge and K. Stüben, Efficient Solution of Finite Difference and Finite Element Equations by Algebraic Multigrid, Multigrid Methods for Integral and Differential Equations (D. J. Paddon and H. Holstein, eds.), The Institute of Mathematics and its Applications Conference Series, Clarendon Press, 1985.

20. E. M. Stein, Harmonic Analysis: Real-Variable Methods, Orthogonality and Oscillatory Integrals, Princeton University Press, 1993. MR1232192 (95c:42002)

21. U. Trottenberg, C. W. Osterlee, and A. Schüller, Multigrid, Appendix A: An Introduction to Algebraic Multigrid by K. Stüben, pp. 413-532, Academic Press, San Diego, 2001.

22. W. L. Wan, T. F. Chan, and B. Smith, An Energy-Minimizing Interpolation for Robust Multigrid Methods, SIAM J. Sci. Comput. 21 (2000), no. 4, 1632-1649. MR1756048(2001a:65162)

23. J. Wloka, Partielle Differentialgleichungen, Teubner, 1982. MR652934 (84a:35002) 
24. J. Xu, Iterative Methods by Space Decomposition and Subspace Correction, SIAM Review 34 (1992), no. 4, 581-613. MR1193013 (93k:65029)

25. Jinchao $\mathrm{Xu}$, Counter examples concerning a weighted $L^{2}$ projection, Mathematics of Computation 57 (1991), 563-568. MR1094965 (92b:65090)

26. P. M. Zeeuw, Matrix-Dependent Prolongations and Restrictions in a Black-Box Multigrid Solver, J. Comput. Appl. Math. 33 (1990), 1-27. MR.1081238 (92c:65152)

27. _ Acceleration of Iterative Methods by Coarse Grid Corrections, Ph.D. thesis, University of Amsterdam, 1997.

Institut für Numerische Simulation, Universität Bonn, Germany

E-mail address: griebel@ins.uni-bonn.de

Institut für Angewandte Mathematik, Universität Bonn, Germany

E-mail address: scherer@iam.uni-bonn.de

Institut für Numerische Simulation, Universität Bonn, Germany

E-mail address: m.a.schweitzer@ins.uni-bonn.de 